\title{
Tactile recognition of raised characters: A parametric study
}

\author{
JACK M. LOOMIS \\ University of California, Santa Barbara, California
}

The accuracy of tactile recognition of raised characters was studied as a function of five variables: (1) height of the upper surfaces of the characters relative to the surrounding base, (2) character set (uppercase roman and braille), (3) spatial extent of the characters (two sizes), (4) mode of touch (static and moving), (5) contact force (two values). All stimuli were felt with the distal pad of the right index finger. The experiment was a repeated-measures design with six subjects. There were significant main effects of all variables except contact force. Of the tests for interaction, only height $\times$ mode was significant; the indication is that, whereas moving the finger permits a considerable improvement in performance over static touch for very shallow characters, moving the finger improves performance only slightly over static touch for characters of much greater height.

Previous investigations of tactile legibility have studied the effects of the typography and spatial extent of raised characters (Johnson \& Phillips, 1981; Loomis, 1981a, 1983; Phillips, Johnson, \& Browne, 1983). The present study sought to determine the effect on legibility of varying the height of the upper surfaces of the characters relative to the surrounding base. Although it would be expected that sufficiently shallow characters would be difficult to sense and thus to identify, the interesting question is whether the variable of character height interacts with other variables such as spatial extent and the mode by which the observer senses the characters. In this experiment, four independent variables were studied in addition to the variable of character height. These were (1) mode of touch (static and moving), (2) type of character (roman and braille), (3) spatial extent of the character (two sizes), and (4) contact force (two values).

\section{METHOD}

\begin{abstract}
Stimuli
The stimuli were raised braille and roman characters produced by photoengraving. The transfer lettering materials used to create the artwork submitted to the photoengraver were the same as in earlier work (Loomis, 1981a, 1982). The artwork was prepared by arranging the 26 uppercase letters and 26 alphabetic braille characters (Sets 2 and 6 from Loomis, 1981a, Figure 1) in two concentric circles. The letters were $5.7 \mathrm{~mm}$ high, and the braille characters were based on a character space that was also $5.7 \mathrm{~mm}$ high (only those characters three dots high filled the vertical extent of the character space). Two negatives were made of the artwork, one of $100 \%$ magnification and the other of $80 \%$ magnification. These two negatives were then used by the photoengraver to make two series of zinc disks, one series with the larger characters (based on $100 \%$ magnification) and the other with the smaller characters (based on $80 \%$ magnification). By virtue of the photoengraving
\end{abstract}

This research was supported by NINCDS Grant NS15129. The author thanks Steven Heimler and Pauline McNulty for their assistance. The author's mailing address is: Department of Psychology, University of California, Santa Barbara, CA 93106. process, characters were $5.7 \mathrm{~mm}$ high (in the plane of the disk) in the $100 \%$ series and $4.6 \mathrm{~mm}$ high in the $80 \%$ series. Within each series, the variable that was manipulated was the etching depth in the direction orthogonal to the plane of the disk; height will henceforth be used to refer to the elevation of the upper surface of the raised characters relative to the etched region of the disk. The heights of 10 characters on each of the 2 corresponding disks (in the $80 \%$ and $100 \%$ series) were measured by micrometer. The measured heights were $0.24,0.39,0.59,0.76$, $1.13,1.40$, and $1.92 \mathrm{~mm}$. These are mean values based on the 20 measurements. The heights within and across corresponding disks were highly uniform; the standard deviations for the different heights averaged $0.035 \mathrm{~mm}$, with the largest being only $0.069 \mathrm{~mm}$ (for the 1.40 $\mathrm{mm}$ height). Because of imperfections in the $1.92-\mathrm{mm}$ disk from the $80 \%$ series, it was not used in the experiment. With its omission, there were 13 disks used in the experiment.

\section{Modes of Touch}

The raised characters were sensed in either of two ways using the right index finger. In the static mode, the subject brought the center of the distal pad into contact with the character, held it there for up to $2 \mathrm{sec}$ without moving, and then withdrew the finger; no lateral motion during contact was permitted. In the moving mode, the subject was permitted to move the finger slightly in lateral, longitudinal, or circular motions while in contact with the character for up to $2 \mathrm{sec}$. These motions were on the order of 1 or $2 \mathrm{~mm}$. It was stressed to the subject that the character was to remain near the center of the distal pad and that at no time during touching was the finger to make contact with only a portion of the character. These restrictions kept the subject from sensing the character with the more sensitive regions close to the fingertip and prohibited sequential scanning of the character. The experimenter monitored the observer's finger during contact to ensure that instructions were followed. The moving mode is the same as the "kinetic" mode used in previous work (Loomis, 1981a, Experiment 1).

\section{Apparatus}

Any stimulus disk could be rotated about its center while suspended horizontally by three equally spaced supports close to its circumference, one of which was a force transducer (Sensotec Model 34). The signal from the transducer was conditioned (Daytronic Model 9170) and then converted to an 8-bit number that made it accessible to software running on an IBM PC. After force calibration for each character set on each of the disks, the computer indicated to the experimenter and subject the incremental force being applied by the finger to the raised character. As one indication, a tone was emitted whenever the incremental force was between 16 and $28 \mathrm{~g}$ for the low-force condition and between 
75 and $125 \mathrm{~g}$ for the high-force condition. Auxiliary apparatus supported the right index finger, hand, and arm comfortably. An adjustable finger guide, positioned just above the disk. was used to keep the finger aligned with the characters.

\section{Subjects}

Six women ranging in age from 21 to 27 years served as paid subjects. None of them had served in a touch experiment previously or was aware of the purpose of the experiment.

\section{Procedure}

Prior to the experiment, each subject received two types of training. First, each was taught, with flashcards and under normal viewing conditions, the alphabetic braille characters. Training continued until no identification errors were made in five successive runs through all 26 cards. Second, each subject trained for $2 \mathrm{~h}$ learning to apply, using each of the modes of touch employed in the experiment, approximately 20 or $100 \mathrm{~g}$ of force to different raised characters. The only feedback to the subject consisted of the tones signaled by the computer whenever the force applied was within the proper range.

Each experimental session of 30-min duration was devoted to one of the 13 disks. The sequences in which the six subjects went through the 13 disks were counterbalanced to minimize sequence effects. In addition, each subject went through the 13 disks in one order and then in the reverse order to provide for some counterbalancing within subjects. Each session consisted of a total of 56 trials, 7 each in the eight conditions obtained by crossing the two types of characters (roman and braille), the two modes of touch (static and moving), and the two values of force $(20$ and $100 \mathrm{~g})$. The computer randomly selected whether the first half of the session would be devoted to letters or to braille. Nested within each half were the two modes of touch, within each of which were nested the two force levels. Selection of the order of conditions and the characters to be presented within each condition was done by sampling with replacement. Before each block of 7 trials, the subject was informed of the condition being tested. On a given trial, the subject began touching the stimulus when ready and was permitted to touch for up to $2 \mathrm{sec}$. If the subject failed to touch in the designated fashion or to apply the proper level of force, the trial was deemed invalid and repeated after reselection of the stimulus. After responding, the subject received feedback about the identity of the character presented.

\section{RESULTS}

The force manipulation had no effect on recognition performance. Averaged over all conditions and subjects, recognition accuracy was $48.5 \%$ correct for the low-force condition and $48.9 \%$ correct for the high-force condition, and examination of the data showed no evidence of an interaction between force and any combination of the other factors. The null effect of force permitted a simplification of the analysis of the remaining four factors (height, size, character, and mode). A four-way repeated-measures analysis of variance (ANOVA) was performed on the data collapsed over the two force conditions. In this analysis, the 1.92-mm-height condition was omitted, since no data were collected for characters in the $80 \%$ magnification condition (4.6-mm vertical extent.)

The $F$ values for all 15 tests of the analysis are given in Table 1. All four of the main effects were significant at the .05 level or beyond; each is readily apparent in Figure 1. Increasing the engraving height from 0.24 to $1.40 \mathrm{~mm}$ increased recognition accuracy from $39.2 \%$ to $54.6 \%$; changing the character size (vertical extent) from 4.6 to $5.7 \mathrm{~mm}$ increased overall recognition accuracy
Table 1

Results of Four-Way Analysis of Variance

\begin{tabular}{lrrrr}
\hline \multicolumn{1}{c}{ Test } & $\mathrm{df}_{1}$ & $\mathrm{df}_{2}$ & \multicolumn{1}{c}{$\mathrm{F}$} & $\mathrm{p}$ \\
\hline $\mathrm{H}$ & 5 & 25 & 26.32 & $<.001$ \\
$\mathrm{~S}$ & 1 & 5 & 85.35 & $<.001$ \\
$\mathrm{C}$ & 1 & 5 & 14.71 & .012 \\
$\mathrm{M}$ & 1 & 5 & 9.27 & .028 \\
$\mathrm{H} \times \mathrm{S}$ & 5 & 25 & .84 & n.s. \\
$\mathrm{H} \times \mathrm{C}$ & 5 & 25 & 1.04 & n.s. \\
$\mathrm{H} \times \mathrm{M}$ & 5 & 25 & 4.93 & .003 \\
$\mathrm{~S} \times \mathrm{C}$ & 1 & 5 & .01 & n.s. \\
$\mathrm{S} \times \mathrm{M}$ & 1 & 5 & 2.65 & n.s. \\
$\mathrm{C} \times \mathrm{M}$ & 1 & 5 & .17 & n.s. \\
$\mathrm{H} \times \mathrm{S} \times \mathrm{C}$ & 5 & 25 & .15 & n.s. \\
$\mathrm{H} \times \mathrm{S} \times \mathrm{M}$ & 5 & 25 & .70 & n.s. \\
$\mathrm{H} \times \mathrm{C} \times \mathrm{M}$ & 5 & 25 & 1.50 & n.s. \\
$\mathrm{S} \times \mathrm{C} \times \mathrm{M}$ & 1 & 5 & .58 & n.s. \\
$\mathrm{H} \times \mathrm{S} \times \mathrm{C} \times \mathrm{M}$ & 5 & 25 & 2.32 & n.s. \\
\hline
\end{tabular}

Note $-H=$ height, $S=$ size, $C=$ character, and $M=$ mode.

from $43.2 \%$ to $53.3 \%$; changing the characters from roman to braille increased recognition accuracy from $43.1 \%$ to $53.5 \%$; and changing the mode from static to moving increased recognition accuracy from $43.3 \%$ to $53.2 \%$. Of the 11 tests for interaction, only height $\times$ mode was significant at the .05 level, but the four-way interaction approached significance $(\mathrm{p}=.073)$.

Post hoc comparisons were made of the effect of mode at each of the six heights used in the preceding analysis. Each comparison was carried out using a repeatedmeasures ANOVA on three factors (mode, size, and character). With $\mathrm{df}_{1}=1$ and $\mathrm{df}_{2}=5$, the following results were obtained. For a height of $0.24 \mathrm{~mm}, \mathrm{~F}=15.47 \mathrm{p}=$ $.011)$; for $0.39 \mathrm{~mm}, \mathrm{~F}=20.32(\mathrm{p}=.007)$; for $0.59 \mathrm{~mm}$, $\mathrm{F}=2.67(\mathrm{p}=.161) ;$ for $0.76 \mathrm{~mm}, \mathrm{~F}=6.46(\mathrm{p}=.051)$; for $1.13 \mathrm{~mm}, \mathrm{~F}=4.70(\mathrm{p}=.081)$; and for $1.40 \mathrm{~mm}$,

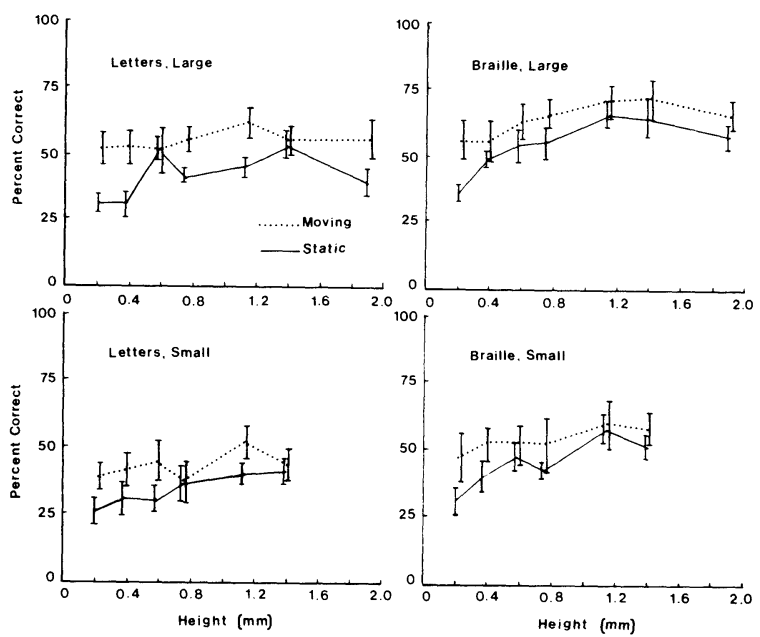

Figure 1. Character recognition accuracy (percent correct) as a function of character height, size (spatial extent), mode, and type of character. The ordinate of each plotted point is the mean value averaged over two force levels and six subjects. The error bars indicate plus and minus one standard error of the mean (across subjects). 
$\mathrm{F}=2.11(\mathrm{p}=.205)$. These results suggest an interpretation of the significant height $\times$ mode interaction in the four-way ANOVA, namely, that the difference between static and moving modes is greatest for the most shallow characters. When one considers the fractional gain in performance in going from static to moving modes as a function of engraving height, this conclusion is even more warranted. When expressed as percentages of the static mode values, the increases in recognition accuracy are $55 \%$ for $0.24 \mathrm{~mm}, 35 \%$ for $0.39 \mathrm{~mm}, 16 \%$ for $0.59 \mathrm{~mm}, 21 \%$ for $0.76 \mathrm{~mm}, 15 \%$, for $1.13 \mathrm{~mm}$, and $10 \%$ for $1.40 \mathrm{~mm}$.

\section{DISCUSSION}

The most interesting finding is the interaction of mode of touch with height of character. It appears that the change from static to moving touch results in the greatest improvement in recognition performance for the most shallow characters. One might interpret this effect in terms of excitation of the slowly adapting (SA) mechanoreceptive afferents of the finger, those most likely involved in the coding of raised characters such as these. Johnson and Lamb (1981) studied the activity of SA units in the monkey finger in response to stimulation by raised braillelike charcacters with heights of 0.7 and $1.2 \mathrm{~mm}$. They found that although SA units responded much more vigorously to moving stimuli than to static stimuli, the reconstructed response patterns of a dense array of SA units did not contain noticeably more information about the moving spatial stimuli than about the static spatial stimuli. One could argue that for characters of sufficient height, static touch results in spatial fidelity of the neural patterns equal to or only slightly inferior to that produced by moving the finger. For very shallow characters, however, it may be that static touch gives rise to considerably less effective excitation of the SA units than does moving touch. Thus, with respect to moving touch, static touch would result in a loss in spatial fidelity in the neural image representing a given character.

The results of the tests for main effects are informative, although not surprising. The significant main effect of height indicates that the more elevated characters are better recognized; most of the improvement in performance can be attributed to static touch over the range of 0.24 to $0.59 \mathrm{~mm} .{ }^{1}$ It stands to reason that since spatial sensitivity must fall off gradually with increasing spatial frequency (Loomis \& Lederman, in press), the more shallow characters lose more of their higher spatial frequencies in the cutaneous processing than do the more elevated characters. Expressed differently, the more shallow characters are effectively more low-pass filtered by the cutaneous processing than are the more elevated characters. Since character size (spatial extent) is a variable that is closely linked to the low-pass filtering of cutaneous processing (see Loomis, 1981a, 1981b; Loomis \& Lederman, in press), character size and height ought to trade off with one another. That is, two different combinations of height and size that result in the same level of recognition performance should correspond to the same effective degree of low-pass filtering.

The significant main effect of mode largely reflects the superiority of the moving mode for the most shallow characters. As the post hoc comparisons indicate, the moving mode did not consistently lead to better performance than did static touch for characters of height $0.59 \mathrm{~mm}$ or greater. The relatively small difference in recognition accuracy between the static and moving modes found here for the more elevated characters is consistent with earlier findings. Loomis (1981a, Experiment 1) studied the same two modes and found that the moving mode gave slightly better performance with characters $1.0 \mathrm{~mm}$ in height (incorrectly reported as $0.8 \mathrm{~mm}$ ); part of the increase with the moving mode, however, may have been due to a sequence effect. Phillips et al. (1983) also compared static touch with a mode similar to the moving mode of the present study and found that permitting movement of the finger (scan mode) improved letter-recognition performance relative to static touch with letters whose surfaces were $1.5 \mathrm{~mm}$ above the surrounding surface. The difference in performance between the two modes was modest, but greater than that found here for characters of large elevation. Part of that larger difference might have been the result of factors other than the introduction of motion. In their scan mode, the subjects were permitted to feel the letters in any way they desired and thus were likely to have used the fingertip, which has better spatial resolution than the center of the distal fingerpad used in the static mode. In addition, the subjects were likely to have tilted their fingers so that only portions of a character were in contact with the finger at any one time. By reducing the degree of lateral masking, this mode of touch makes possible the sensing of detail not perceptible with static touch (Loomis, 1981b; Loomis \& ApkarianStielau, 1976; Loomis \& Lederman, in press).

The main effects of character size and type corroborate the findings of earlier studies (Loomis, 1981a, 1982; Johnson \& Phillips, 1981; Phillips et al., 1983). Of more interest is the fact that these variables do not interact with each other or with the other variables studied in the experiment. The absence of interactions simplifies the understanding of how these variables influence tactile perception and will prove useful in attempts to develop formal models of tactile character recognition.

That contact force results in no main effect and does not interact with the other variables indicates that touching with roughly $20 \mathrm{~g}$ of force and touching with roughly $100 \mathrm{~g}$ of force provide about the same information to the subject. This conclusion cannot be extrapolated to the entire range of contact forces, for surely performance would have declined had forces on the order of $1 \mathrm{~g}$ or less been used. Incidentally, the 5to 10-g contact forces reported in earlier work (Loomis, 1981a, 1982) were in error, for an improved measurement procedure indicates that subjects use forces averaging about $50 \mathrm{~g}$ ( $\mathrm{SD}=14 \mathrm{~g}$ across subjects) when they sense raised characters with the moving mode of touch used here.

\section{REFERENCES}

Johnson, K. O., \& LAMB, G. D. (1981). Neural mechanisms of spatial tactile discrimination: Neural patterns evoked by braille-like dot patterns in the monkey. Journal of Physiology, 310, 117-144.

Johnson, K. O., \& PhILlips, J. R. (1981). Tactile spatial resolution: I. Two-point discrimination, gap detection, grating resolution, and letter recognition. Journal of Neurophysiology, 46, 1177-1191.

Loomis, J. M. (1981a). On the tangibility of letters and braille. Perception \& Psychophysics, 29, 37-46.

LoomIs, J. M. (1981b). Tactile pattern perception. Perception, 10, 5-27.

Loomis, J. M. (1982). Analysis of tactile and visual confusion matrices. Perception \& Psychophysics, 31, 41-52.

Loомis, J. M. (1983, November). Tactile and visual legibility of seven character sets. Paper presented at the meeting of The Psychonomic Society, San Diego, CA.

Loomis, J. M., \& APKarian-Stielau, P. (1976). A lateral masking effect in tactile and blurred visual letter recognition. Perception \& Psychophysics, 20, 221-226.

LoOmis, J. M., \& LEDERMAN, S. J. (in press). Tactual perception. In K. Boff, L. Kaufman, \& J. Thomas (Eds.), Handbook of perception and human performance. New York: Wiley.

Nolan, C. Y., \& Kederis, C. J. (1969). Perceptual factors in braille word recognition. New York: American Foundation for the Blind.

Phillips, J. R., Johnson, K. O., \& Browne, H. M. (1983). A comparison of visual and two modes of tactual letter resolution. Perception \& Psychophysics, 34, 243-249.

\section{NOTE}

1. The production standard for braille specifies a dot height of $0.43 \mathrm{~mm}$ (Nolan \& Kederis, 1969). Since there was relatively little effect of height with the moving mode, which is similar to the normal mode of reading braille, the present results indicate that little gain in legibility would be realized by increasing the height of braille dots.

(Manuscript received for publication August 31, 1984.) 